\title{
Supraclavicular lymph node involvement in endometrial carcinoma, rare site of metastasis: A case report
}

\author{
Trisha Arora ${ }^{1}$, Naina Kumar ${ }^{2, *}$ \\ ${ }^{1}$ Post Graduate Student, ${ }^{2}$ Associate Professor, Dept. of Obstetrics and Gynaecology, Maharishi Markandeshwar Institute of \\ Medical Sciences and Research, Haryana, India
}

*Corresponding Author:

Email: drnainakumar@gmail.com

Received: $22^{\text {nd }}$ June, 2018

Accepted: $7^{\text {th }}$ August, 2018

\begin{abstract}
Endometrial carcinoma is one of the commonest gynecological cancers of female genital tract, usually occurring after menopause. Supraclavicular lymph node is one of the rare sites of metastasis in the endometrial carcinoma. The present article illustrates the typical and atypical metastasis, surgical staging and role of lymph node dissection in patients with endometrial carcinoma. Typical sites are pelvic, the para-aortic nodes, the peritoneum, and lungs. Atypical sites are extra-abdominal lymph nodes, brain, bones, liver, soft tissue and adrenals. It is therefore important for the radiologists to recognize typical \& atypical sites of metastases seen in patients of endometrial carcinoma so as to facilitate early diagnosis and treatment.
\end{abstract}

Keywords: Endometrial carcinoma, Lymph node, Metastasis, Menopause.

\section{Introduction}

Endometrial carcinoma is amongst the commonest gynecological malignancies of the female genital tract $\&$ accounts for about $6 \%$ of all the cancers in women and approximately $2 \%$ of cancer deaths occurring annually all over the world. This disease is primarily seen in menopausal and post-menopausal females having peak incidence in 55-65 years aged women. $75 \%$ of patients are $50 \mathrm{yrs}$ and older and around $5 \%$ are younger than $40 \mathrm{yrs} .{ }^{1}$ Significant most prognostic factors are histological type \& grade, depth of involvement of myometrium, lymphovascular invasion \& lymph node status. ${ }^{2}$ Endometrial carcinoma is further divided into several histological categories on the basis of their cell type. Endometrioid being the most common cell type, accounts for majority of the cases, (70-80\%) and is subdivided into grade 1 (well differentiated) to grade 3 (poorly differentiated). ${ }^{3}$ Other aggressive pathologic variants with a high risk of metastasis include clear cell carcinoma, squamous cell carcinoma, papillary serous carcinoma, mixed and undifferentiated types. ${ }^{4}$

Common sites of metastasis of endometrial carcinoma include pelvic, para-aortic lymphnodes, peritoneum, vagina \& lungs. Atypical sites are intraabdominal organs, bones, abdominal wall, brain, muscle. Extra abdominal occurrence is seen rarerly (0.4-1\%) which include supraclavicular lymph node, mediastinal and axillary lymph nodes. ${ }^{5}$

Radical surgery has an important role for management of the patients having locally or regionally advanced endometrial carcinoma, especially if it is utilized along with the adjuvant radiation or chemotherapy. The standard surgery for women having advanced stage endometrial cancer involves panhysterectomy with pelvic, para-aortic lymphadenectomy and if it is possible then resection of entire visible tumor.

We report a case of 60 years old female having complaint of postmenopausal bleeding, later diagnosed as endometrial carcinoma stage III C1with supraclavicular lymph node involvement.

\section{Case Report}

A 60 years old postmenopausal female presented to the gynecological outpatient department of a rural tertiary care centre of Northern India with the complaints of heavy postmenopausal bleeding per vaginum since 3 months, for which she was previously taking some treatment from a local private hospital, but got no relief. On general examination the patient was calm, conscious, well oriented to time place and person and her general condition was within normal limits. Physical examination revealed a palpable left sided supraclavicular lymph node measuring around $3 \times 4 \mathrm{~cm}$. On abdominal examination no organomegaly was noted. On pelvic examination uterus was 6-8 weeks size, anteverted and anteflexed, mobile, bilateral fornix free. Her whole abdomen and pelvic ultrasound revealed bulky uterus with pelvic lymph node enlargement. This was further confirmed by Magnetic Resonance Imaging, which showed large endometrial mass with myometrial invasion and multiple pelvic lymphadenopathy (FIGO stage IIIC) (Fig. 1). The patient was posted for fractional curettage, which further confirmed the finding of endometrial carcinoma and revealed endometrioid (type I) adenocarcinoma of endometrium. At the same time FNAC was planned from the left sided enlarged supraclavicular lymph node which also showed metastatic deposits (Fig. 2). After all investigations and written informed consent, the patient was taken for total abdominal hysterectomy 
with bilateral salpingo-oophrectomy and pelvic lymphadenectomy. Left sided common iliac and external iliac chain removed, a matted lymph node of $5 X 4 \mathrm{~cm}$ present over right external iliac vein, artery and extending up to common iliac vein removed and sent for histopathology. (Fig. 3)

On gross examination (Fig. 4) endometrial cavity was filled with large grey brown mass arising from endometrial cavity measuring $5 \times 2.5 \times 1.7 \mathrm{cms}$. Depth of tumour invasion into myometrium was $17 \mathrm{~mm}$ and thickness of myometrium was $20 \mathrm{~mm}$. Tumour extended from fundus to lower uterine segment sparing cervix. Right common, external, and internal iliac lymph nodes show nodal parenchyma completely replaced by tumour. Microscopically, the tumor was confirmed to be endometroid adenocarcinoma (Fig. 5) with wide spread necrosis (stage III C1) with lymph node involvement. Post-operatively the patient was put on chemotherapy Carboplatin AUC $420 \mathrm{mg} / \mathrm{m}^{2}$ and Pactitaxel $240 \mathrm{mg} / \mathrm{m}^{2}$ three weekly. Patient is responding well to the treatment and frequently reports for follow up.

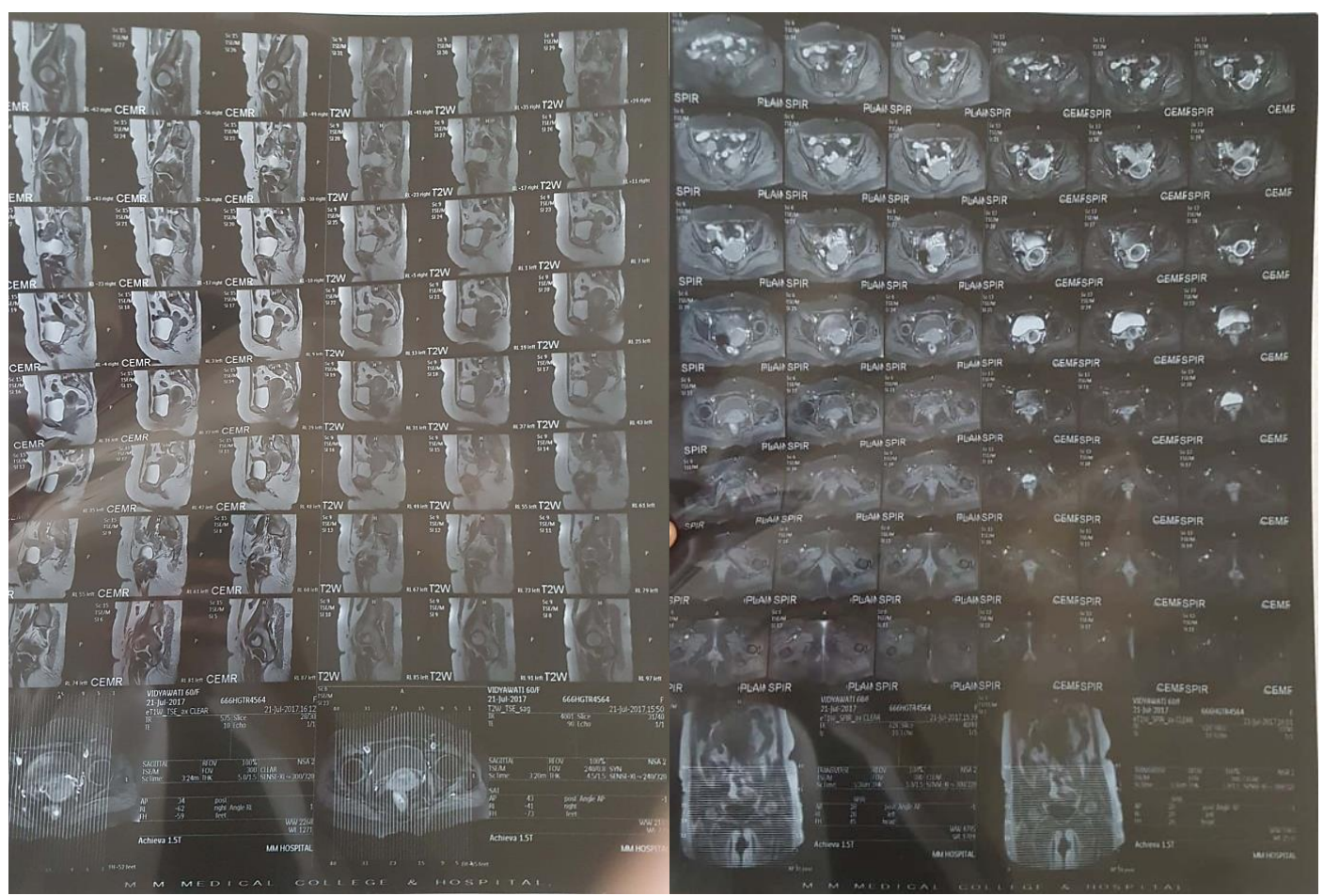

Fig. 1: MRI showing large endometrial mass with myometrial invasion and right pelvic lymphadenopathy

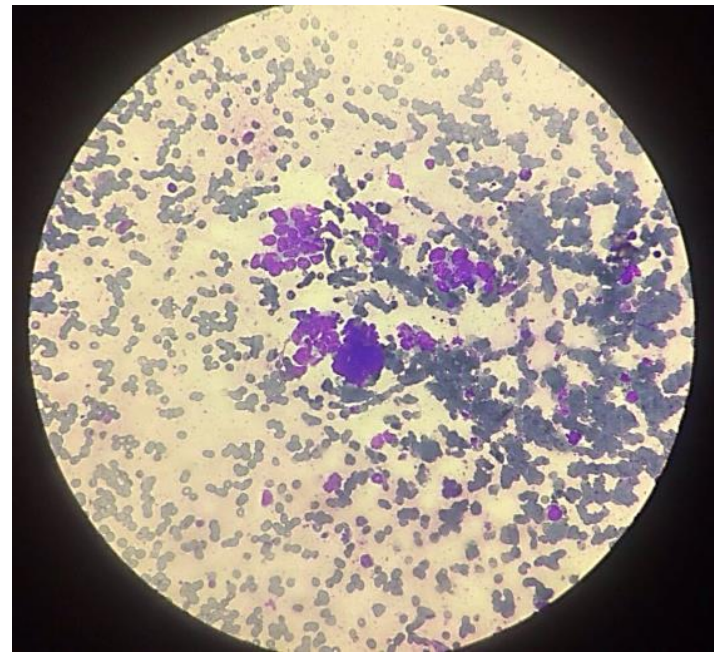

Fig. 2: FNAC of supraclavicular lymph node showing malignant cells forming glandular pattern. May-Grünwald Giemsa, magnification 400 x

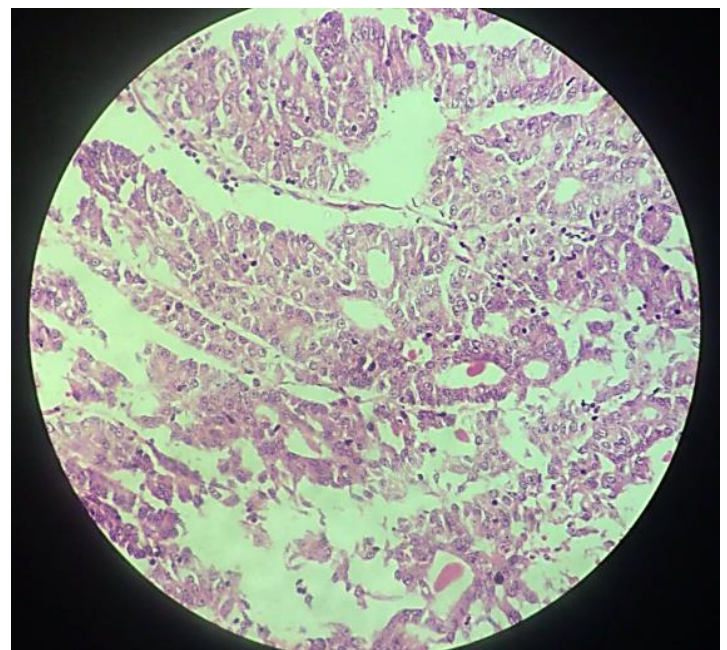

Fig. 3: Right common, external and internal iliac (matted) lymph node involved by metastasis showing a confluent glandular 


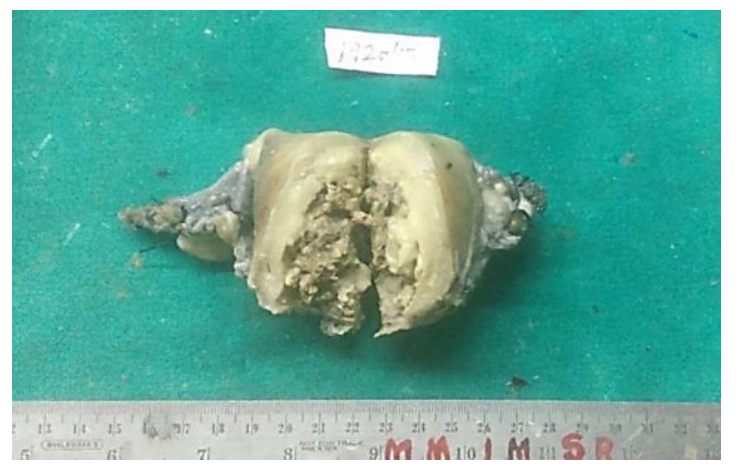

Fig. 4: Gross examination of endometrial cavity showing large grey brown mass

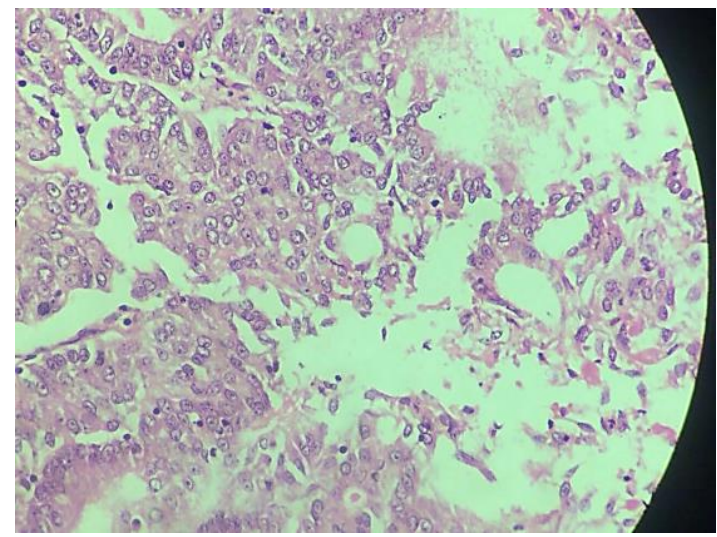

Fig. 5: Endometriod adenocarcinoma; tumor cells having vascular nuclei exhibiting moderate to marked anisonucleosis, prominent nucleoli with brisk mitosis. Haematoxylin and eosin stain, magnification $400 x$

\section{Discussion}

In developed countries endometrial carcinoma is most frequently seen malignancy of female genital tract and is the second commonest in developing countries. ${ }^{6}$

Vaginal bleeding being commonly associated with the presence of disease, fortunately majority of the patients are therefore diagnosed at an early stage which results in overall favourable prognosis with high cancer specific survival rates. ${ }^{7,8}$ Pelvic lymph nodes is common site of extra-uterine disease in patients with clinical early stage disease and thus pelvic lymph node dissection is an integral part of standard surgical procedures. $^{6}$

The 3 common spread patterns in endometrial carcinoma are through local invasion, lymphatic spread $\&$ hematogenous spread. The lymphatic drainage of the uterus normally occurs through the parametrium to the pelvic sidewalls including spread to the iliac and obturator nodes. Metastatic disease may then spread from the pelvic sidewall to the common iliac and then para-aortic nodes. The alternative drainage, including the uterine fundus, may also occur along the ovarian vessels directly to the higher para-aortic nodes. ${ }^{9}$ Supraclavicular lymph node involvement is uncommon.
The mainstay of treatment in women having endometrial cancer is surgery involving total hysterectomy with bilateral salpingo-oophorectomy, also pelvic and para-aortic lymphadenectomy and if possible then resection of entire visible tumor. Some include routine omental sampling, peritoneal staging biopsies to define the extent of disease, specially is those with a serous histological subtype followed by post operative adjuvant therapy. ${ }^{10}$

The ACOG committee has suggested that the 'adjuvant therapy' should only be limited to patients having positive nodes. ${ }^{11}$ Theoretically, there are several potential advantages of lymphnode removal. Complete surgical staging helps to identify the patients with documented lymphatic dissemination, thus targeting postoperative treatment. Although main aim of lymphadenectomy is documenting the presence of lymphatic metastases, there is no consensus about the best adjuvant approach patients having positive lymph nodes. The Gynecologic Oncology Group 122 trial $\underline{50}$ had suggested that chemotherapy (doxorubicin and cisplatin) provides a better survival than the radiotherapy (whole abdominal irradiation) in stages III or IV and with $\leq 2 \mathrm{~cm}$ of residual disease ${ }^{11}$ However, chemotherapy has decreased distant recurrence rate (19$10 \%$ ) at a cost of higher pelvic recurrence rate that is 13 to $18 \%$, whereas post operative radiotherapy helps in reducing local pelvic recurrence as it is administered to the pelvic region. The target volume consists of previous site of uterus, adnexa, parametria, proximal two-thirds of vagina and lymphatic drainage regions along the internal iliac vessels up to the promontory. ${ }^{8}$ Patients who are not put on postoperative radiotherapy must be followed closely, especially in the first $3 \mathrm{yrs}$, to diagnose and to treat a loco-regional recurrence at the earliest stage possible.

\section{Conclusion}

Endometrial carcinoma is although most frequent malignancy seen in women, supraclavicular lymph node involvement is rare which is an evidence of widespread dissemination, thus depicting poor prognosis for these patients and should therefore be diagnosed at the earliest possible for better overall survival.

\section{References}

1. Lachiri B, Lazrak I, Ibrahim A. et al. Endometrial Adenocarcinoma in a 26 year old Woman: Case report. ISSR Journal. 2014;9:808-811.

2. Vidal F, Rafii A. Lymph node assessment in endometrial cancer: towards personalized medicine. Obstet Gynecol Int. 892465;2013

3. Bansal N, Yendluri V, Wenham RM. The molecular biology of endometrial cancers and the implications for pathogenesis, classification, and targeted therapies. Cancer Control. 2009;16:8-13. 
4. Kurra V, Krajewski KM, Jagannathan J, Giardino A, Berlin S, Ramaiya N. Typical and atypical metastatic sites of recurrent endometrial carcinoma. Cancer Imaging. 2013;13:113-22.

5. Kitajima, K., Murakami, K., Yamasake, E. Performance of FDG-PET/CT in the diagnosis of recurrent endometrial cancer. Ann Nucl Med. 2008;22:103-109.

6. Bezerra, A.L., Batista, T.P., Martins, M.R., Carneiro, V.C. Surgical treatment of clinically early-stage endometrial carcinoma without systematic lymphadenectomy. Rev Assoc Med Bras. 2014;60:571576.

7. Benedet, J.L., Bender, H., Jones, H. III, Ngan, H.Y. \& Pecorelli, S.FIGO staging classifications and clinical practice guidelines in the management of gynecologic cancers. FIGO Committee on Gynecologic Oncology. Int J Gynaecol Obstet. 2000;70:209-262.

8. Creutzberg, C.L., Van Utten, W.L.J., Koper, P.C.M. et al, Surgery and postoperative radiotherapy versus surgery alone for patients with stage-1 endometrial carcinoma: multicentre randomized trial. Lancet. 2000;355:14011411.
9. Devaja O., Wells T., Sentinel Lymph Node Biopsy in Endometrial Cancer (Scientific Impact Paper No. 51). Rcog. 2017.

10. Rauh-Hain, J.A., Del Carmen, M.G. Treatment for advanced and recurrent endometrial carcinoma: combined modalities. Oncologist. 2010;15:852-861.

11. Bogani, G., Dowdy, S.C., Cliby, W.A., Ghezzi, F., Rossetti, D., Mariani, A. Role of pelvic and para-aortic lymphadenectomy in endometrial cancer: current evidence. J Obstet. Gynaecol. Res. 2014;40:301-311.

How to cite this article: Arora T, Kumar N. Supraclavicular lymph node involvement in endometrial carcinoma, rare site of metastasis: A case report. Ind $\mathrm{J}$ Obstet Gynecol Res. 2018;5(3):438-441. 